\title{
Numerical simulations of global instability in separated flows at high Mach number
}

\begin{tabular}{|r|l|}
\hline Journal: & 2012 New Orleans Conferences \\
\hline Manuscript ID: & Draft \\
\hline luMeetingID: & 2231 \\
\hline Date Submitted by the Author: & n/a \\
\hline Contact Author: & Zhang, Kangping \\
\hline
\end{tabular}

\section{SCHOLARONE ${ }^{\text {m }}$ \\ Manuscripts}




\title{
Numerical simulations of global instability in separated flows at high Mach number
}

\author{
Kangping Zhang*, Neil D. Sandhamªnd Zhiwei $\mathrm{Hu}^{\ddagger}$ \\ AFM Research Group, University of Southampton, Southampton SO17 1BJ, UK
}

\begin{abstract}
The global stability or instability of supersonic ramp flow and of a jet in supersonic crossflow is investigated. The three-dimensional Navier-Stokes equations are solved directly with no model applied. Ramp flow is studied at Mach number 4.8 and Reynolds numbers 6,843 and 3,422, based on inflow boundary layer displacement thickness and freestream velocity. The laminar base flows are stable in two-dimensions. Simulations in three-dimensions show that the high Reynolds number case is globally unstable, while the flow is stable at the lower Reynolds number, suggesting that a critical Reynolds number for instability exists between these two Reynolds numbers. A spanwise wavelength of 12 times the incoming boundary layer displacement thickness is found to be the most unstable for the ramp flow with Reynolds number of $\mathbf{6 , 8 4 3}$. Similar conclusions are found for a sonic jet injected into a Mach 6.7 crossflow, which is stable at low jet momentum flux ratios $\left(J_{p}\right)$, but becomes globally unstable as $J_{p}$ increases. Streamwise vortices are observed in the unstable jet cases and a spanwise wavelength of 8 times the incoming boundary layer displacement thickness is found to be the preferred mode of global instability.
\end{abstract}

\section{Introduction}

In high speed flow, shock-wave/boundary-layer interaction (SWBLI) can lead to flow separation and significantly affect the aerothermodynamic loads, such as wall pressure, skin friction and heat transfer. The problem has been studied for more than 60 years, ${ }^{1}$ however some important flow phenomena still cannot be clearly explained or predicted, such as unsteady pressure loading caused by flow unsteadiness. ${ }^{1-3}$ Configurations of ramp flow and shock wave impingement with laminar inflow conditions have been used to study the instability of high speed SWBLI, which requires high resolution of the flowfield. So far both large eddy simulation (LES) $)^{4,5}$ and direct numerical simulation (DNS $)^{3,6-11}$ have been used. For the shock wave impingement flow, Pagella ${ }^{8}$ and Yao et al. ${ }^{10}$ compared linear stability theory (LST) and the parabolized stability equations (PSE) approach with DNS, respectively. There were regions where both methods were in accord with the DNS results. By comparing the density flowfield of both ramp flow and shock wave impingement, Pagella et al. ${ }^{9}$ found that the effect of a two-dimensional (2D) impinging shock and that of a compression ramp on laminar boundary layer were practically identical when the impinging shock angle was set equal to half the ramp angle. However there might be differences between a three-dimensional (3D) compression ramp and a 3D impinging shock for non-linear cases. ${ }^{9}$ Robinet $^{2}$ showed flow with shock wave impingement became 3D and unsteady starting from the incident shock angle and the spanwise extension. He suggested that the physical origin for the $3 \mathrm{D}$ behaviour was a 3D stationary global instability, and the present contribution continues with this approach for ramp and jet flow.

Both experimental and numerical studies have been carried out for hypersonic laminar ramp flow. Chanetz et al. ${ }^{12}$ performed an experimental study of 2D ramp flow at Mach 10 and then compared the performance of Navier-Stokes (NS) and Direct Simulation Monte Carlo (DSMC) solvers by simulating the experimental configuration. The conclusion was that DMCS solvers performed less well than solvers based on the Navier-Stokes equations. Based on Chanetz's work, Marini ${ }^{7}$ studied the effect of different parameters on ramp flow with Navier-Stokes solvers and showed that ramp angle, wall temperature assumption

\footnotetext{
*PhD student, Faculty of Engineering and the Environment, k.zhang@soton.ac.uk.

${ }^{\dagger}$ Professor, Faculty of Engineering and the Environment.

$\ddagger$ Lecturer, Faculty of Engineering and the Environment.
} 
and geometric configuration were the main factors for mechanical and thermal loads, as well as separation characteristics over the ramp. Lüdeke and Sandham ${ }^{11}$ studied ramp flow with Mach number 4.8 for several Reynolds numbers. Secondary recirculation zones were found at a Reynolds number of 3,422. It was clearly demonstrated that the length of the separation zone increases with increasing ramp angle and Reynolds number. They observed a convective transition process in a 3D ramp flow which was extended in the spanwise direction from a laminar 2D flow.

Early research on jets in supersonic crossflow used experimental methods and mainly focused on the flowfield around the injection port. Cubbison et al. ${ }^{13}$ and Kaufman $\mathrm{II}^{14}$ noticed an increase in both upstream and downstream surface pressure caused by the interaction between the jet and the laminar incoming flow. Cubbison et al. ${ }^{13}$ found that Mach number and jet pressure ratio, rather than Reynolds number, were the two main factors on the pressures distributions and levels. There was also theoretical analysis done to determine the 2D flowfield near the injection port based on inviscid flow and a perfect gas assumption. ${ }^{15}$

To provide experimental data for numerical analysis, Powrie ${ }^{16}$ did extensive experiments on 2D and 3D laminar hypersonic jet interactions for flat plates in a Mach 6.69 stream and obtained data on surface pressure, separation length, as well as flow visualisations. For a 2D slot jet, Powrie found that the separation length is determined by the momentum flux ratio $\left(J_{p}\right)$ which is defined using stagnation pressure of the jet $\left(p_{0_{J}}\right)$, the slot width $(w)$, the free stream pressure $\left(p_{\infty}\right)$ and the distance from the slot centre to the leading edge of the flat plate $\left(L_{s}\right)$ as

$$
J_{p}=\frac{p_{0_{J}} w}{p_{\infty} L_{s}}
$$

Roberts et al. ${ }^{17}$ used the same facility as Powrie to quantify the heat transfer using thermochromic liquid crystals. The boundary layer growing on the plate ahead of the jet was also laminar. It was found the heat fluxes were up to several times the undisturbed laminar flow along lines of flow reattachment. Numerical work based on Powrie's 2D slot case was first carried out by Qin and Redlich. ${ }^{18}$ They successfully predicted the massively separated upstream interaction region with good agreement with experimental observations. This agreement showed that laminar flows dominated the upstream interaction, although the simulation was limited to $2 \mathrm{D}$ steady flow. Gajbhiye ${ }^{19}$ simulated a sonic jet in supersonic crossflow and compared with Powrie's experiments. It was found that the flow became unsteady when the momentum flux ratio $\left(J_{p}\right)$ was increased to 0.4 and widening of the slot jet made the separation point move further upstream. Ilak $^{20}$ studied instability of round jet in crossflow at low values of the velocity inflow ratio by DNS. Results showed self-sustained oscillation developed in shear layer downstream of the jet which was also the source of instability.

The present paper investigates the instability of ramp flow and of a jet in crossflow at high Mach numbers. The objective is to see whether the global modes of instability, found by Robinet ${ }^{2}$ for the shock impinging case, are also present for ramp or jet-in-crossflow configuration. The paper is organized as: Section II briefly describes the numerical tools and the simulation setup. Results are discussed in Section III, followed by concluding remarks.

\section{Numerical methods and simulation setup}

\section{A. Numerical methods}

The simulations presented in this paper solve the unsteady, 3D compressible Navier-Stokes equations in Cartesian coordinates $\left(x_{i}, \mathrm{i}=1,2,3\right)$. The governing equations can be written in the following non-dimensional form

$$
\begin{gathered}
\frac{\partial \rho}{\partial t}+\frac{\partial\left(\rho u_{i}\right)}{\partial x_{i}}=0 \\
\frac{\partial\left(\rho u_{i}\right)}{\partial t}+\frac{\partial\left(\rho u_{i} u_{j}\right)}{\partial x_{j}}=-\frac{\partial p}{\partial x_{i}}+\frac{\partial \tau_{i j}}{\partial x_{j}} \\
\frac{\partial(\rho E)}{\partial t}+\frac{\partial(\rho E+p) u_{i}}{\partial x_{i}}=-\frac{\partial q_{i}}{\partial x_{i}}+\frac{\partial\left(u_{i} \tau_{i j}\right)}{\partial x_{j}},
\end{gathered}
$$

where $\tau_{i j}$ and $q_{i}$ are the non-dimensional viscous stress tensor and heat conduction vector components respectively, which are defined as 


$$
\tau_{i j}=\frac{\mu}{R e}\left(\frac{\partial u_{i}}{\partial x_{j}}+\frac{\partial u_{j}}{\partial x_{i}}-\frac{2}{3} \delta_{i j} \frac{\partial u_{i}}{\partial x_{i}}\right) \quad q_{i}=-\frac{\mu}{R e(\gamma-1) M_{\infty}^{2} \operatorname{Pr}} \frac{\partial T}{\partial x_{i}} .
$$

Expression of non-dimensional dynamic viscosity $\mu$ defined by Sutherland's law, as well as the Reynolds number $R e$ and Prandtl number $P r$, are given by

$$
\mu=T^{\frac{3}{2}} \frac{1+S^{*} / T_{r e f}^{*}}{T+S^{*} / T_{r e f}^{*}} \quad \quad R e=\rho_{\infty}^{*} u_{\infty}^{*} L^{*} / \mu_{\infty}^{*} \quad \operatorname{Pr}=c_{p}^{*} \mu^{*} / k^{*},
$$

where $S^{*}$ is the Sutherland temperature $(110.4 \mathrm{~K})$ and $T_{r e f}^{*}$ is a reference free stream temperature. An asterisk is used to denote dimensional quantities. All variables are non-dimensionalized with reference quantities. The reference length $L^{*}$ is specified to be the inflow boundary layer displacement thickness $\delta_{0}^{*}$. The reference velocity $U_{\infty}^{*}$, density $\rho_{\infty}^{*}$, temperature $T_{\infty}^{*}$ and viscosity $\mu_{\infty}^{*}$ all use the freestream values. All results are presented in non-dimensional form in this paper.

A fourth-order central difference scheme is employed for spatial discretization at internal points. The stable boundary treatment proposed by Carpenter et al. ${ }^{21}$ is applied to points close to boundaries, which guarantees overall fourth-order spatial accuracy. Time marching is performed using the low-storage thirdorder explicit compact Runge-Kutta method of Wray. ${ }^{22}$ The entropy splitting method from Sandham et al. ${ }^{23}$ is applied to the non-linear terms. It splits the inviscid flux into conservative and non-conservative parts for stability improvement. For shock wave capture, TVD scheme of Yee et al. ${ }^{24}$ with an Ducros sensor ${ }^{25}$ which only switches on TVD outside the boundary layer is used. The artificial compression method (ACM) by Harten $^{26}$ is also included in the code.

\section{B. Simulation setup}

Flow over a $12^{\circ}$ compression ramp is simulated at a freestream Mach number of 4.8 and static temperature of $55.4 \mathrm{~K}$. The Reynolds numbers used here are 3,422 and 6,843 based on the inflow boundary layer displacement thickness. The wall is considered to be isothermal with temperature equal to the laminar adiabatic value.

The computational domain extends 215.2 and 325 (non-dimensional) before and after the ramp corner respectively. It has a height of 50 in the wall-normal direction. The domain is meshed with stretched grids in both directions to better resolve the flow near the wall and the corner as shown in Figure 1 . Threedimensional meshes are extruded from the $2 \mathrm{D}$ mesh with different spanwise lengths $\left(L_{z}\right)$ ranging from 3 to 24. Following an earlier grid study, spanwise grid point $N_{z}=16$ is chosen for current study.

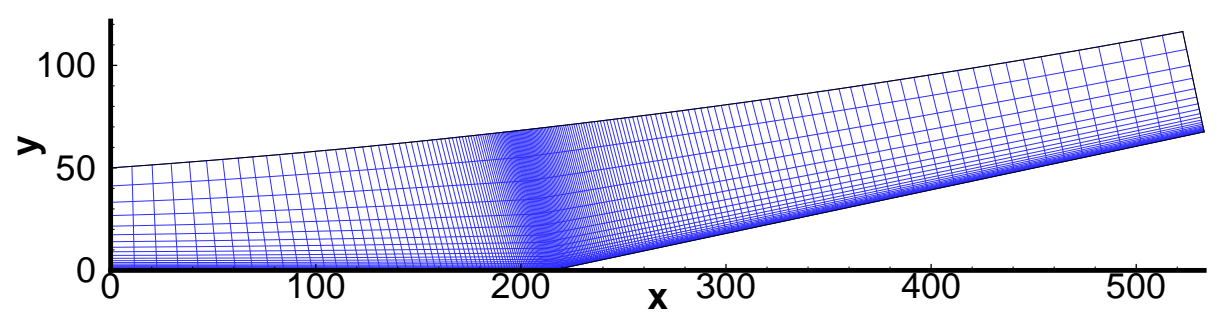

Figure 1. 2D mesh for $12^{\circ}$ ramp study (the gridlines are displayed every 10 points).

For the jet in crossflow case, the freestream Mach number is 6.69 with a Reynolds number of 2,500 and static temperature of $63.11 \mathrm{~K}$, while the wall temperature is set to $298 \mathrm{~K}$ to match the conditions of the Powrie $^{16}$ experiments. Sonic air with temperature of $244.17 \mathrm{~K}$ is injected from a slot of width 3.8 at $x=113$ on the bottom wall where a mixed boundary condition is applied. A rectangular computational domain of $160 \times 80$ (or $200 \times 80$ for $3 \mathrm{D}$ baseflow and instability studies with the downstream domain elongated) is used. The mesh is stretched in both the streamwise and wall-normal directions to obtain a finer mesh near the wall and slot area as shown in Figure 2. The 3D mesh is extruded from the 2D mesh with different $L_{z}$, ranging form 3 to 64 . For all $3 \mathrm{D}$ cases 8 spanwise grid points are adopted following a study that compared $N_{z}=8$, 16 and 32 with simpler meshes, showing $N_{z}=8$ is sufficient to resolve the exponential stage of growth and get the same growth rate of $3 \mathrm{D}$ instability.

For both flow, the similarity solution for a compressible laminar boundary layer ${ }^{27}$ is used to generate the inflow profiles and initialize the flow field. The solutions are then marched in time. Extrapolation and 


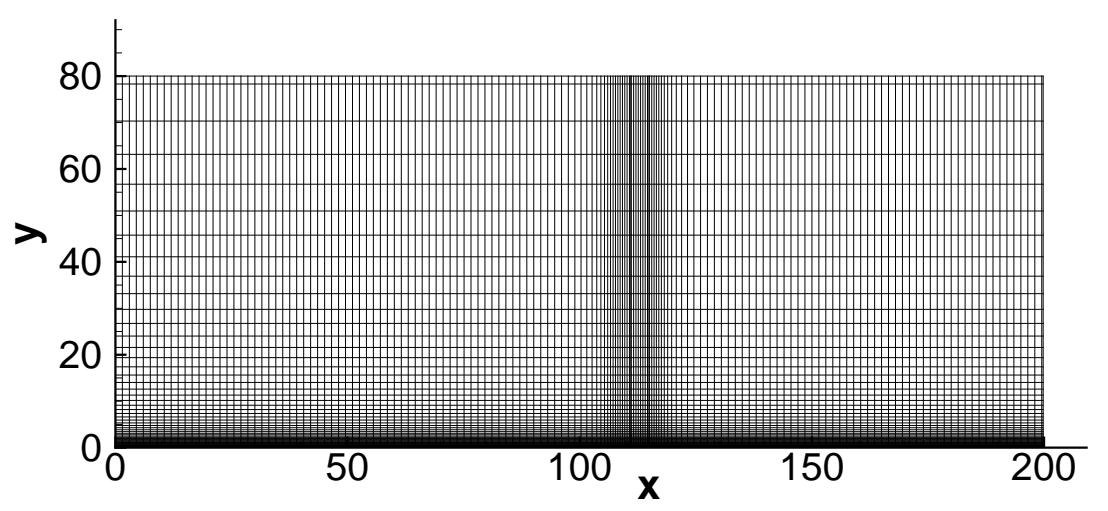

Figure 2. 2D mesh for jet in crossflow (Mesh is displayed every other 10).

characteristic boundary conditions are used at inflow and outflow respectively. A no-slip wall is adopted, while an integral characteristic condition is used for the jet inflow and the upper boundary. Periodic boundary conditions are applied in the $z$ direction. For the jet in crossflow, the jet profile is defined at $y=0$ with a parameter $\delta$ which decides the slopes of jet velocity and temperature at the edge of the slot with specified centre-line values in the following form

$$
\begin{array}{ll}
\text { for }\left(L_{s}-w / 2\right)<x \leq L_{s}: \begin{array}{l}
v=v_{j} \tanh \left(\frac{x-\left(L_{s}-w / 2\right)}{\delta}\right) \\
T=T_{w}+\left(T_{j}-T_{w}\right) \tanh \left(\frac{x-\left(L_{s}-w / 2\right)}{\delta}\right),
\end{array} \\
\text { for } L_{s}<x \leq\left(L_{s}+w / 2\right): \begin{array}{l}
v=-v_{j} \tanh \left(\frac{x-\left(L_{s}+w / 2\right)}{\delta}\right) \\
T=T_{w}+\left(T_{w}-T_{j}\right) \tanh \left(\frac{x-\left(L_{s}+w / 2\right)}{\delta}\right),
\end{array}
\end{array}
$$

where $x$ is the non-dimensional x coordinate, $v_{j}$ and $T_{j}$ are velocity in wall normal direction and temperature at centre-line of jet, respectively and $w$ is the slot width, set $w / 2=1.9 . T_{w}$ is the wall temperature. $\delta$ of 0.0717 is obtained by iteration to achieve $99 \%$ of centre-line value within $5 \%$ of the slot width and the profiles are smoother when a larger $\delta$ is used.

\section{Results}

\section{A. Ramp flow}

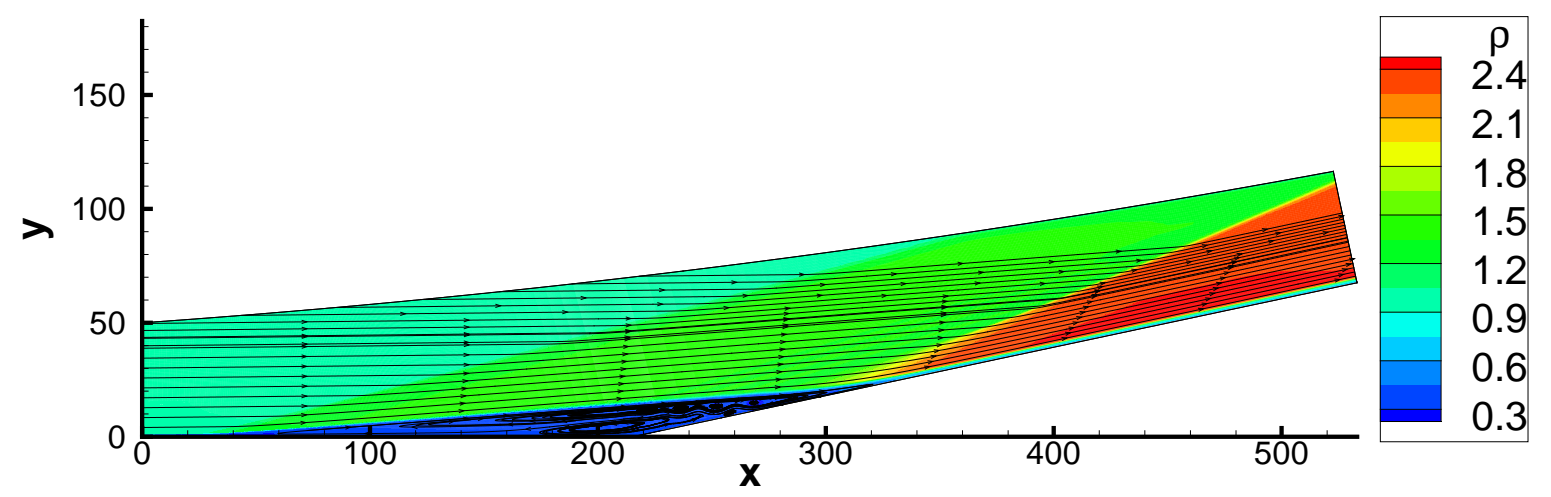

Figure 3. Density $(\rho)$ contours for a typical ramp flowfield superposed by streamlines. 
As expected for a compression ramp with high speed incoming flow, a shock wave is formed after the corner. The interaction between the shock wave and boundary layer induces flow separation, causing separation shock as shown in Figure 3. In this section, simulations are first run for $2 \mathrm{D}$ configurations to validate the code and get steady grid-independent results for further $3 \mathrm{D}$ simulations. Code validation was done by comparing the skin friction coefficient $\left(C_{f}\right)$ distribution, plotted as a function of local Reynolds number $R_{x}$ $\left(R_{x}=\sqrt{R e_{x}}\right)$ with the result of Lüdeke and Sandham ${ }^{11}$ for a ramp angle of 6 degrees and $R e=3,422$. Lüdeke and Sandham ran the case with two Navier-Stokes solvers (Flower and SBLI). Both of them agreed with reference data, with only small differences between two solvers due to the different discretization methods. ${ }^{11}$ As seen on Figure 4, good agreement is also observed between the current simulation (Test case in the figure) and the SBLI result obtained by Lüdeke and Sandham, ${ }^{11}$ and with the published result of Pagella et al. ${ }^{9}$

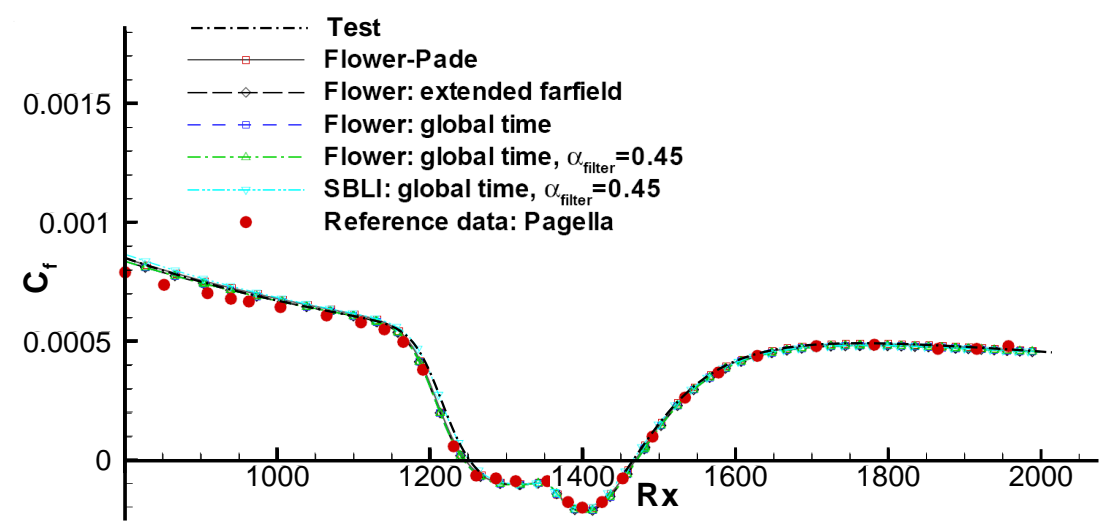

Figure 4. Comparison of skin friction distributions with data from Pagella et al. ${ }^{9}$ and Lüdeke and Sandham. ${ }^{11}$

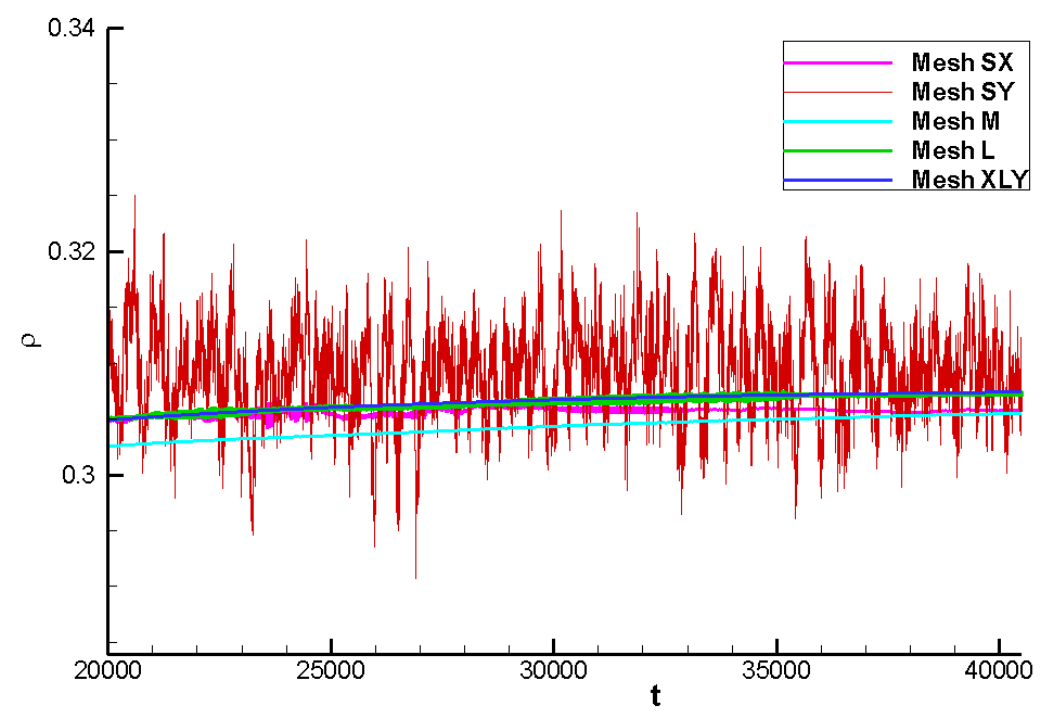

Figure 5. Time history of $\rho$ at $(x=196.8, y=6.7)$ for 2D ramp flow.

To check the grid-dependency, simulations based on five meshes with different grid distributions in the streamwise or wall-normal direction (meshes SX: 800*169, SY: 1153*109, M: 1153*169, L: $1800^{*} 250$ and XLY: $\left.1800^{*} 376\right)$ were carried out. The ramp angle used here is increased to $12^{\circ}$ and the Reynolds number to 6,843 . Density time histories at a monitor point $(x=196.8, y=6.7)$ just upstream of the ramp are plotted in Figure 5. Significant difference can be seen between result from meshes SY and M. The result 
from mesh SY is unsteady with amplitude about 0.03 , while the flowfield from mesh M, as well as meshes $\mathrm{SX}, \mathrm{L}$ and XLY, is steady. The simulation is more sensitive to the mesh in the wall-normal direction and the difference between results of meshes $\mathrm{M}$ and $\mathrm{L}$ is caused by the different number of grid points in the wall-normal direction. According to the agreement of results from mesh L and mesh XLY, results from mesh L are considered to be mesh-independent. The same conclusion was obtained from two statistical measures of pressure, the mean pressure and root-mean-square (RMS) of pressure on the wall, as plotted in Figure 6 . It is shown that the mesh in the wall-normal direction is the principal factor for the average length of separation deduced from the distribution of the mean pressure. Figure 6(b) shows that the flow is steady when a finer mesh is used. Figure 7 shows $C_{f}$ distributions from different meshes. The oscillation region near the corner for mesh SY is caused by the lack of grid resolution in the wall normal direction, while the downstream oscillation for mesh SX is caused by a lack of grid points downstream. Results from mesh L and mesh XLY agree both upstream and downstream. All of the comparisons mentioned above lead to the conclusion that results from mesh $\mathrm{L}$ are mesh-independent and reliable.

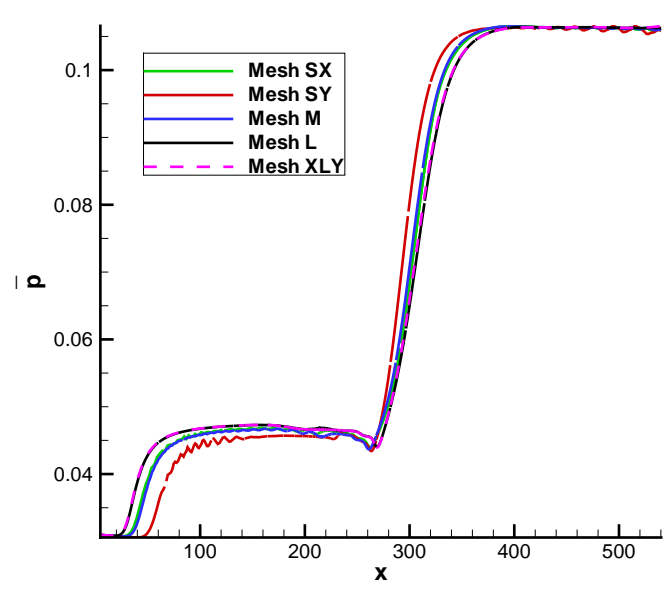

(a)

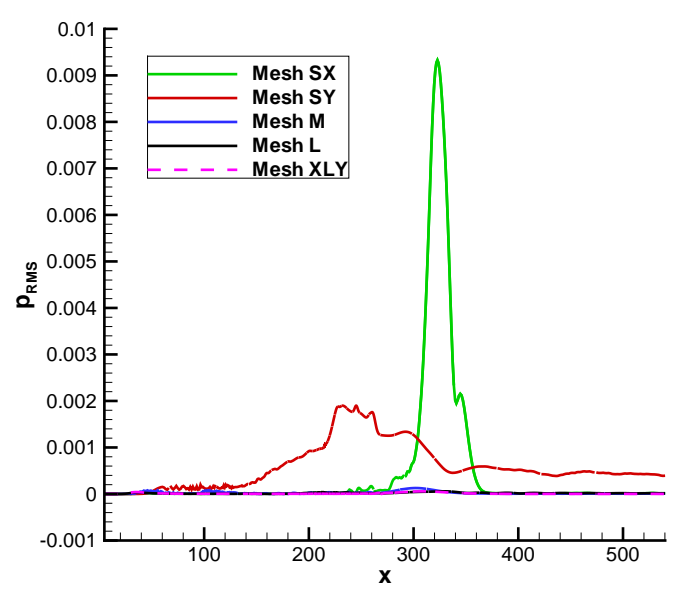

(b)

Figure 6. Statistic values of pressure on the wall: (a)mean pressure, (b)root-mean-square of pressure.

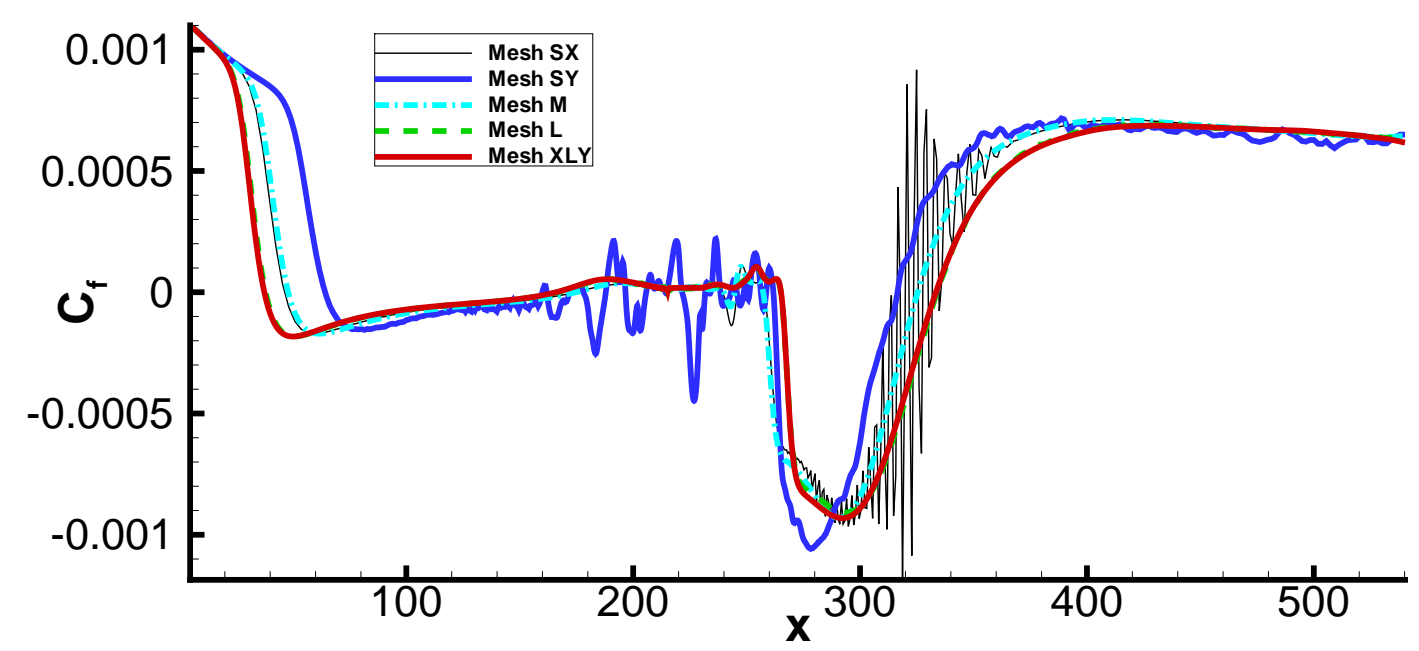

Figure 7. Skin friction distributions for different meshes. 
Flow fields for two different Reynolds numbers are shown in Figure 8. Recirculation zones are clearly seen. The length of the secondary recirculation zone at the corner increases from 45 to 110 as the Reynolds number is increased from 3,422 to 6,843 .

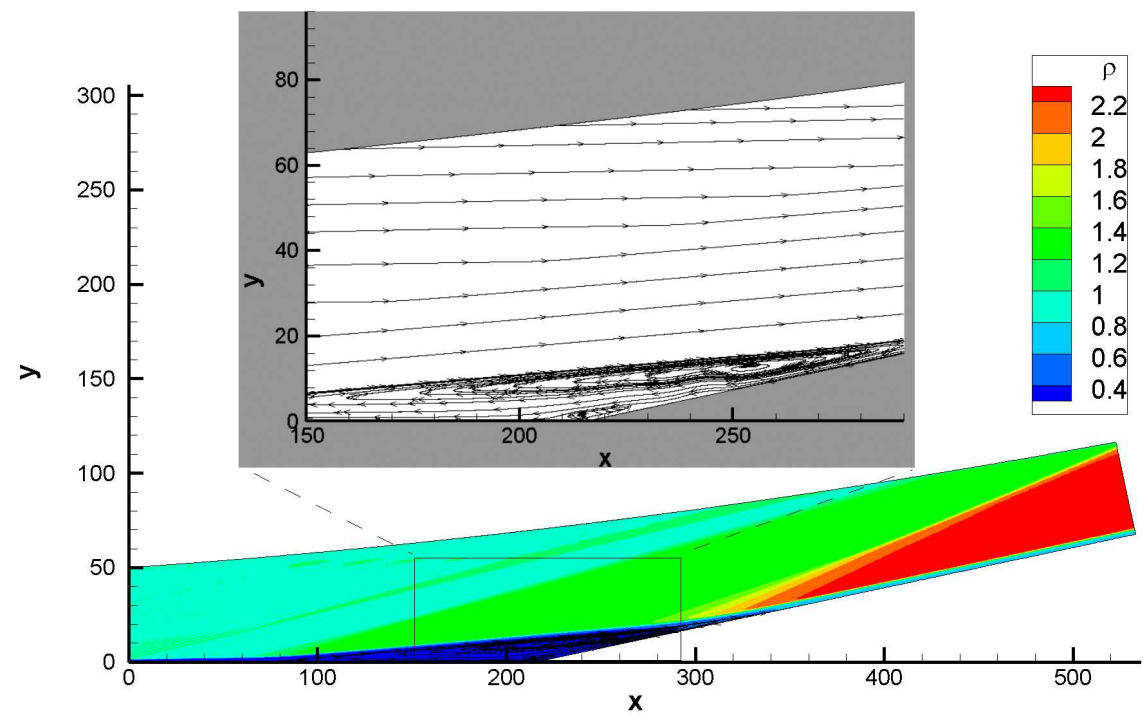

(a)

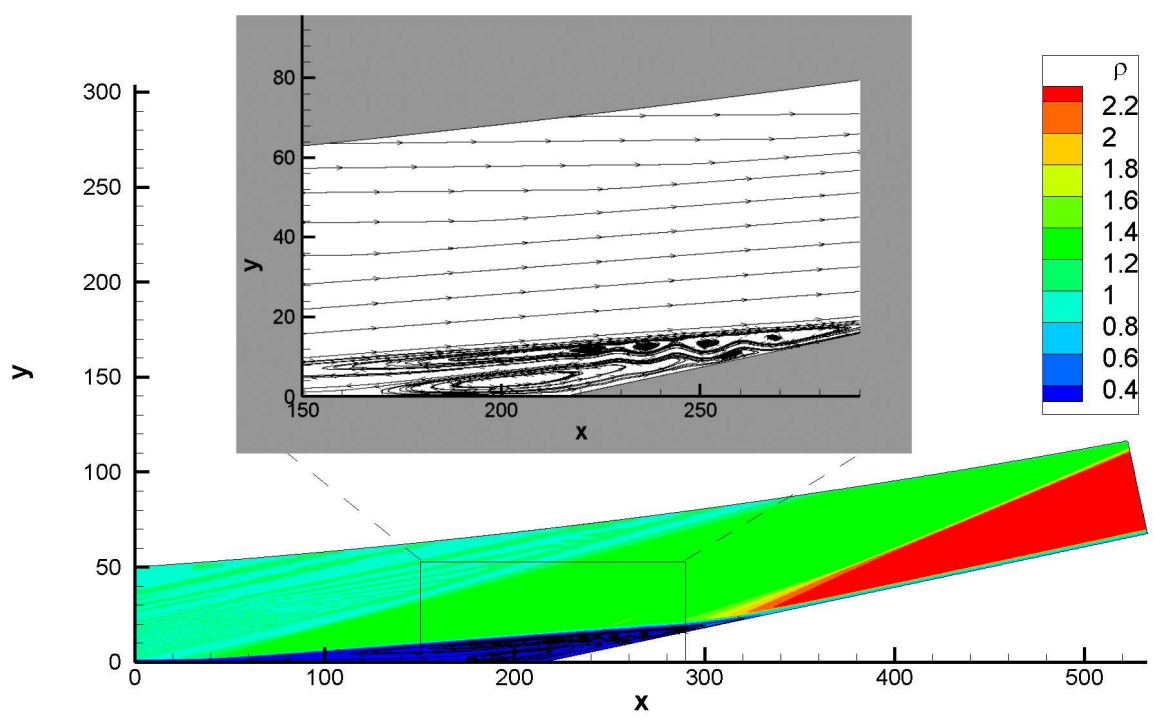

(b)

Figure 8. The separated flow for the $12^{\circ}$ ramp at two different Reynolds numbers (a) $3,422,(b) 6,843$.

Three-dimensional $12^{\circ}$ ramp flows are calculated based on the steady $2 \mathrm{D}$ results for a Reynolds number of 6843.3 with a spanwise length $L_{z}=12$ and number of spanwise grid points $N_{z}=16$. The initial flow field is formed by extending the converged $2 \mathrm{D}$ results in the spanwise direction. Small perturbations are then added to the spanwise velocity component to study the instability of the ramp flow, which has been observed from Figure 6 to be stable in 2D when sufficiently fine grids are used. Perturbations are added to a small region in the recirculation zone. The perturbation has a Gaussian profile in the $x-y$ plane and varies sinusoidally in the spanwise direction, given by

$$
w^{\prime}(x, y, z)=A e^{-\frac{1}{4}\left[\left(x-x_{0}\right)^{2}+\left(y-y_{0}\right)^{2}\right]} \sin \left(\frac{2 \pi z}{L_{z}}\right)
$$


where $A$ is the amplitude, given a value of $A=3.5 \times 10^{-12}$. For the added perturbation, the centre of the Gaussian profile, $\left(x_{0}, y_{0}\right)$, is chosen right above the ramp corner with coordinates $(x=234.7, y=10.7)$. A parameter $\varepsilon(x, y)$, measuring the mean square fluctuation of the spanwise velocity at three monitor points, is used to monitor the development of the $3 \mathrm{D}$ flow instability. The parameter is defined by

$$
\varepsilon(x, y)=<w(x, y, z)^{2}>-<w(x, y, z)>^{2}
$$

where $<.>$ denotes an average in the spanwise direction. Simulations are then run for 3D flow for the spanwise length $L_{z}$ equal to $2,3,6$ and 24. The same number of grid points $N_{z}=16$ is used for all cases as only a single sine wave is added in the spawnwise direction.

Figure 9 compares the growth of the spanwise variation in time at different Reynolds numbers for ramp flows with $L_{z}=12$. It can be seen that the spanwise variation stays at a very low level for the low Reynolds number case $(R e=3,422)$. For the high Reynolds number case $(R e=6,843)$ exponential growth is seen. It appears that there is a threshold in Reynolds number, which lies between the two studies cases, for the 3D global instability to develop for ramp flow.

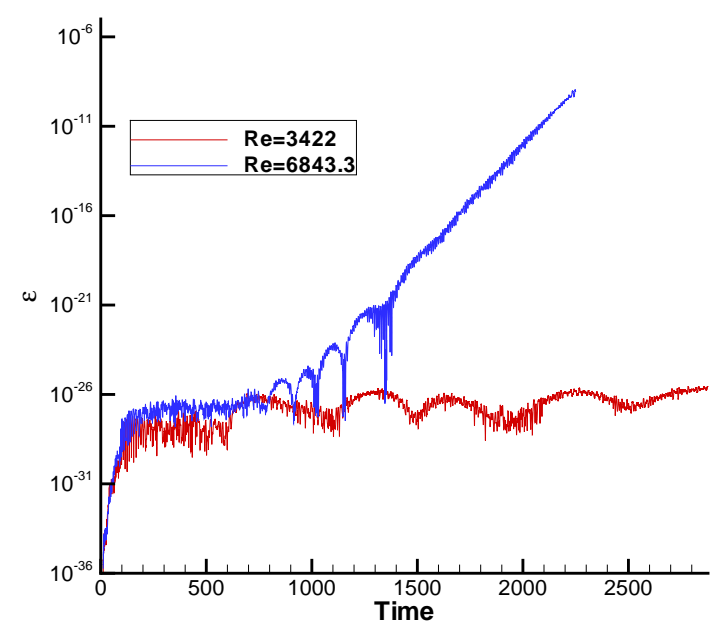

Figure 9. Growth of $\epsilon$ with time at different Reynolds numbers for the same configuration.

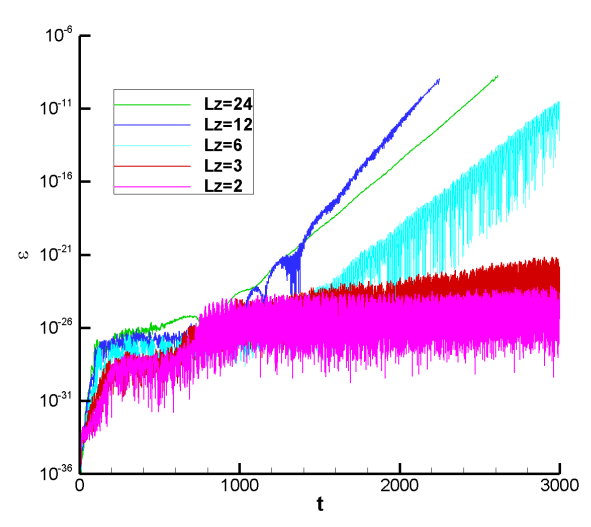

(a)

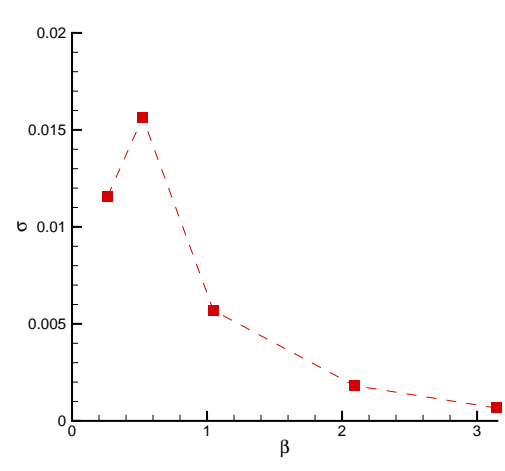

(b)

Figure 10. Statistical measure of instability for ramp flow with different spanwise lengths: (a) variation of $\varepsilon$, defined by (9) at monitor point along with time, (b) growth rate of spanwise variation with wavenumber. 
It is found that the growth rate varies with the wavelength of the added perturbation. Figure 10(a) shows growth of the spanwise variations with time. By measuring the slopes in the exponential stage, a plot of the growth rate $\sigma=0.5 d(\ln \varepsilon) / d t$ as a function of the wavenumber $\beta=2 \pi / L z$, is obtained as shown in Figure 10(b). The peak of the curve in Figure 10(b) corresponds to $L_{z}=12$. In Figure 10(a), the oscillations appearing for $L_{z}=2,3$ and 6 are dependent on the locations of the monitor point relative to the large recirculation zones.

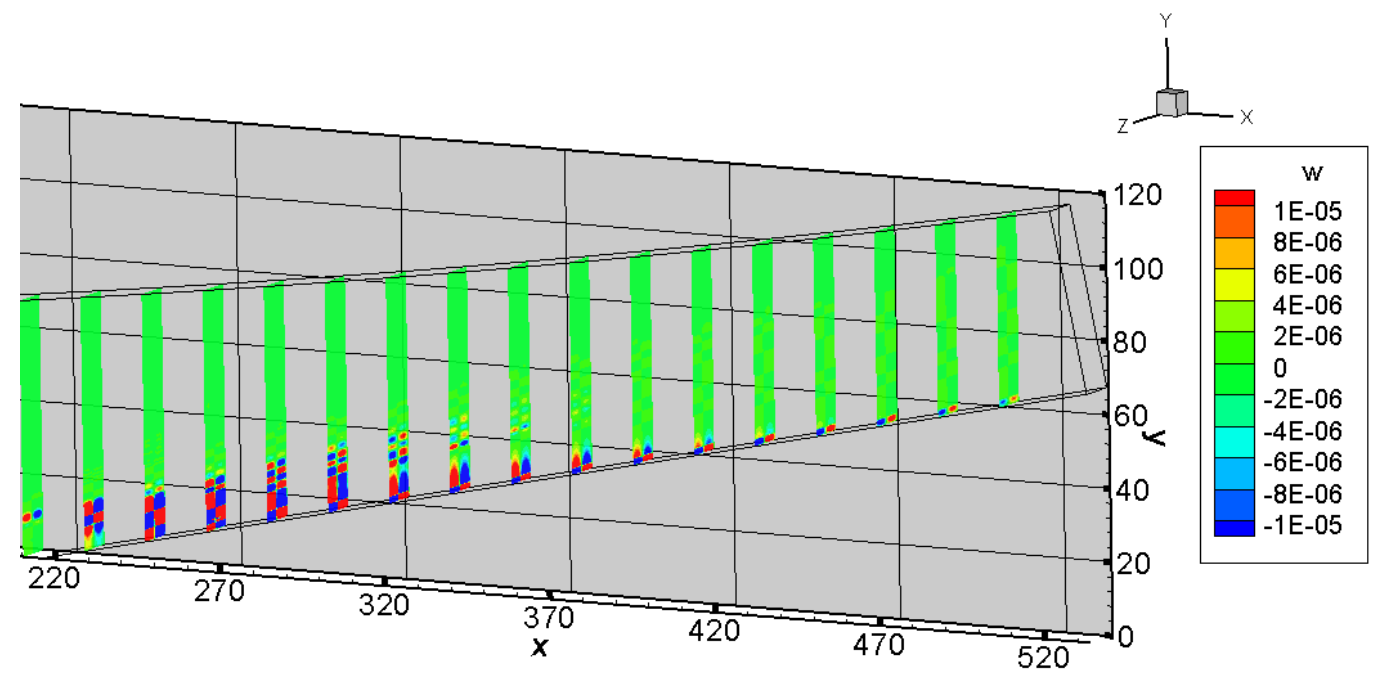

Figure 11. Spanwise variation of $w$ visualized on different $y-z$ planes.

In the exponential growth stage, the development of the added perturbation in the flowfield is shown in Figure 11. Both upstream and downstream $w$-velocity distributions show a single-wave in the $z$ direction, with the highest amplitudes seen in the region $x=220$ to 300 and $y=0$ to 20 which corresponds to the secondary recirculation zone.

\section{B. Jet in crossflow}

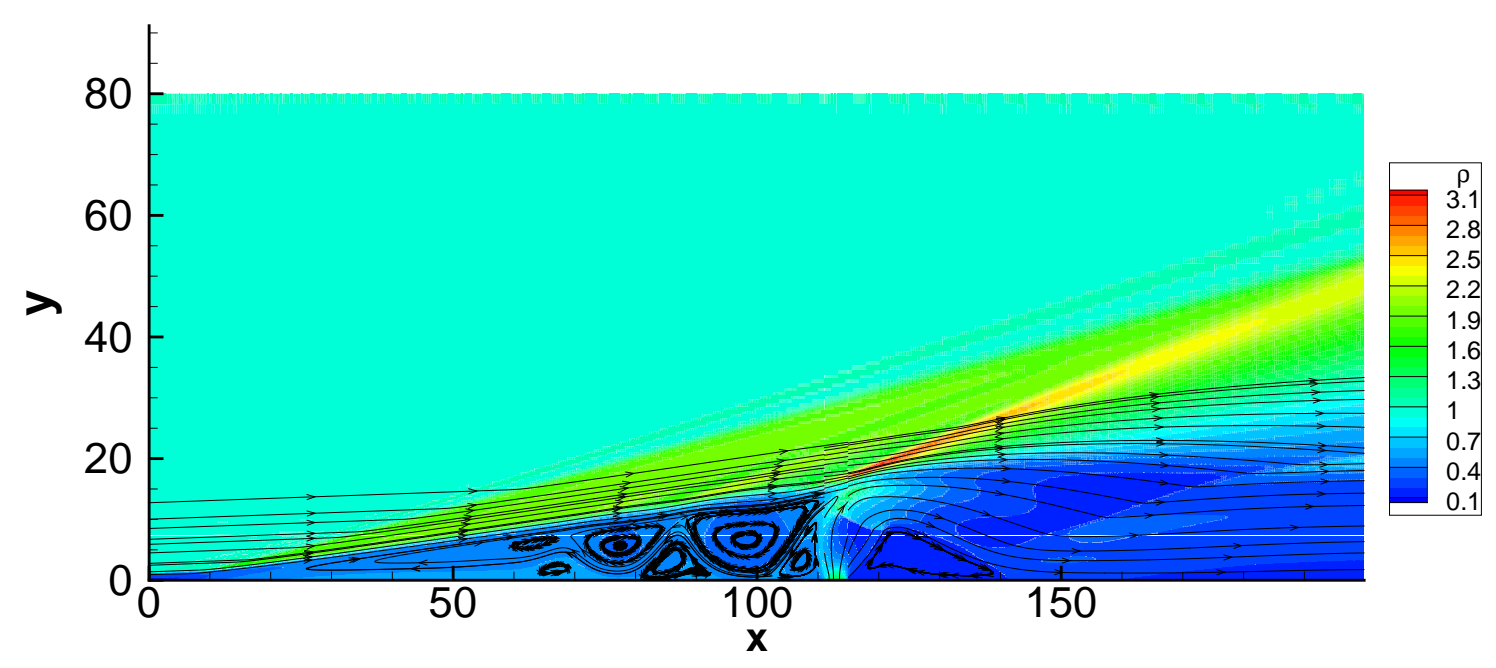

Figure 12. Density $(\rho)$ distribution for 2D jet in crossflow flowfield superimposed with streamlines. 
The flowfield of the 2D jet in crossflow is shown in Figure 12 by contours of density with superposed streamlines. Both upstream and downstream flow separations are formed by the flow injection, generating an upstream separation shock and shear layer, a bow shock above the jet as well as a recompression shock downstream.

Gajbhiye $^{19}$ showed the upstream separation length had a close relationship with the jet profile and $J_{p}$. Figure 13 shows distribution of mean wall pressure $(\bar{p})$ for $J_{p}$ of $0.1,0.2,0.3$ and 0.4 but the same $\delta$ of 0.0717 . Differences between the curves are mainly seen in the amplitude of $\bar{p}$ at the jet (since the jet pressure is directly proportional to $J_{p}$ ), its variation around the jet and the place where $\bar{p}$ starts to increase, i.e. the upstream separation. It is observed that $\bar{p}$ shows more variations near the jet and the separation appears closer to the inflow boundary with higher $J_{p}$. For the jet flow, a larger pressure means larger mass flow rate, producing larger recirculation zones. To make sure the separation zone is not affected by the inflow boundary, $J_{p}=0.3$ is used in the study.

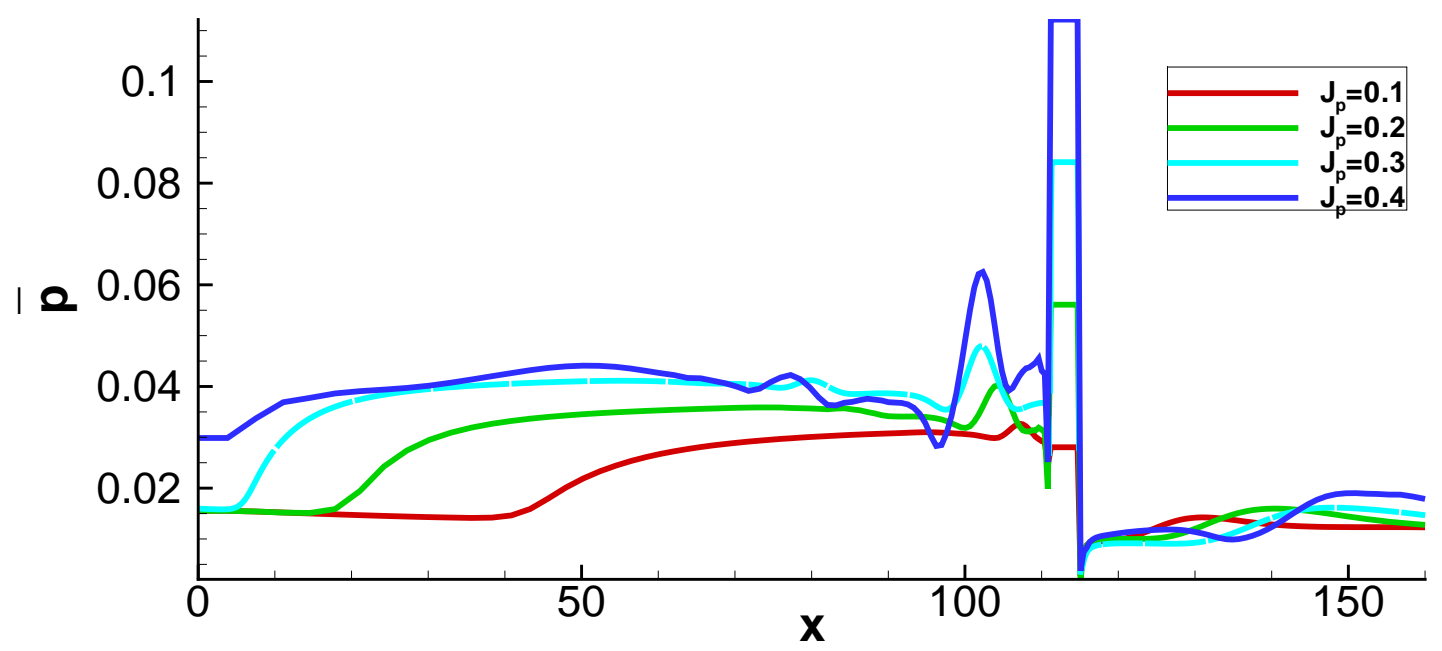

Figure 13. Distributions of mean wall pressure $(\bar{p})$ with different $J_{p}$.

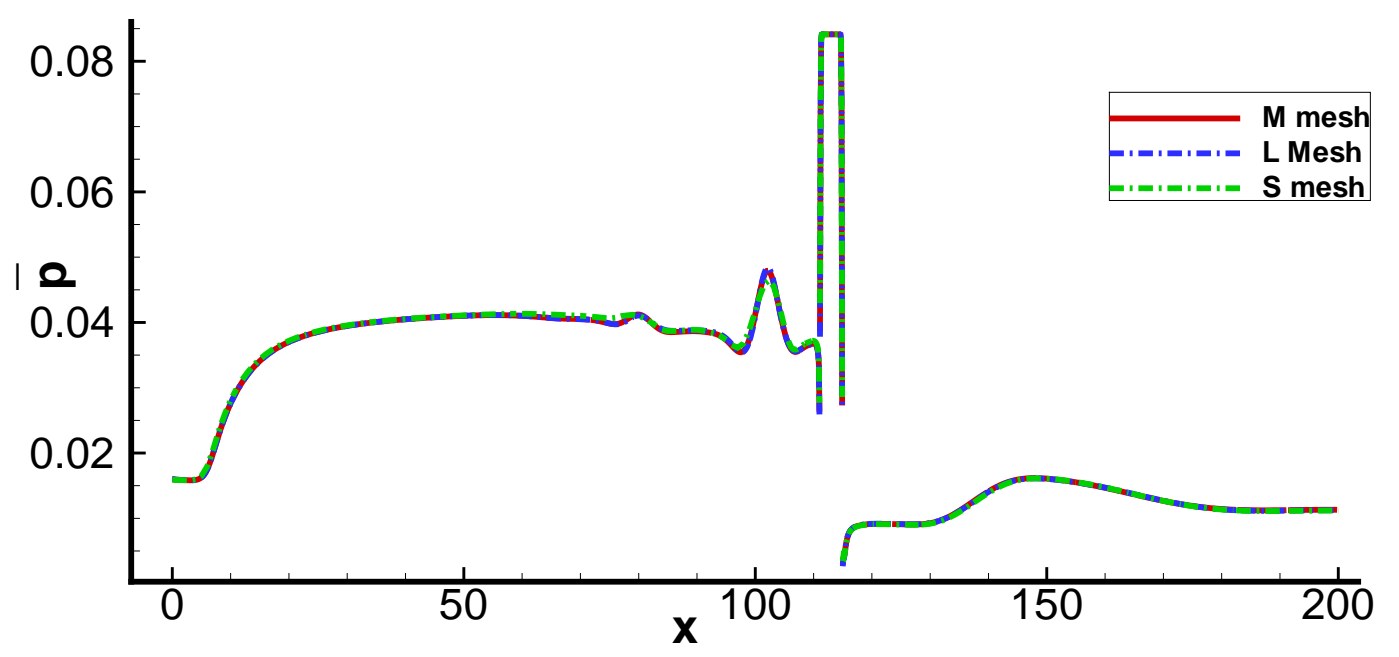

Figure 14. Distribution of $\bar{p}$ for different meshes (the gaps in the curves are caused by interface of different computational blocks). 
A mesh dependency study is carried out using three different meshes with different streamwise resolution, denoted as an S mesh (379*256), M mesh (765*256) and L mesh (1088*256). Meshes with an even finer resolution in the wall normal direction or around the jet area were also tried, but led to small amplitude unsteadiness in the 2D simulation. To get a clean observation of global instability development and eliminate the interference, the resolution in the wall normal direction and around the jet in streamwise direction were limited to $N_{y}=256$ and $\Delta x=0.1$, respectively and the Ducros sensor was turned off. Nevertheless, there are still always more than 65 grid points within boundary layer in the wall normal direction. Densities from the $\mathrm{S}, \mathrm{M}$ and $\mathrm{L}$ mesh setups at three different monitor points, lying upstream, above the jet and downstream, follow the same trend to become steady, though there are variations with time before a non-dimensional time of 10,000. The differences of mean densities from the S, M and L meshes at the same point are within $0.5 \%$ after steady flow is achieved, so it can be concluded that flowfields obtained from different meshes tend to the same steady flow. Small differences in distributions of $\bar{p}$ from different meshes can be seen between results on the $\mathrm{S}$ mesh and other meshes, as shown in Figure 14. They are mainly focused on the recirculation zone. The overlap of results from $M$ mesh and $L$ mesh leads to conclusion that results from the $M$ mesh are grid-independent.

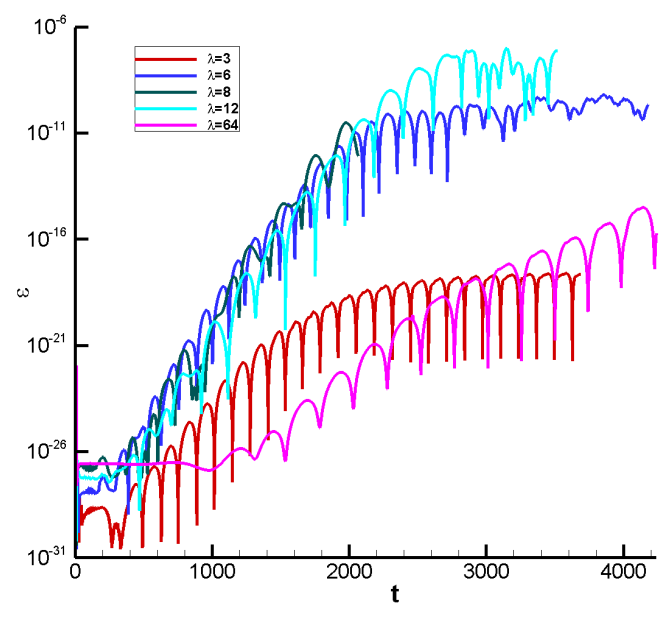

(a)

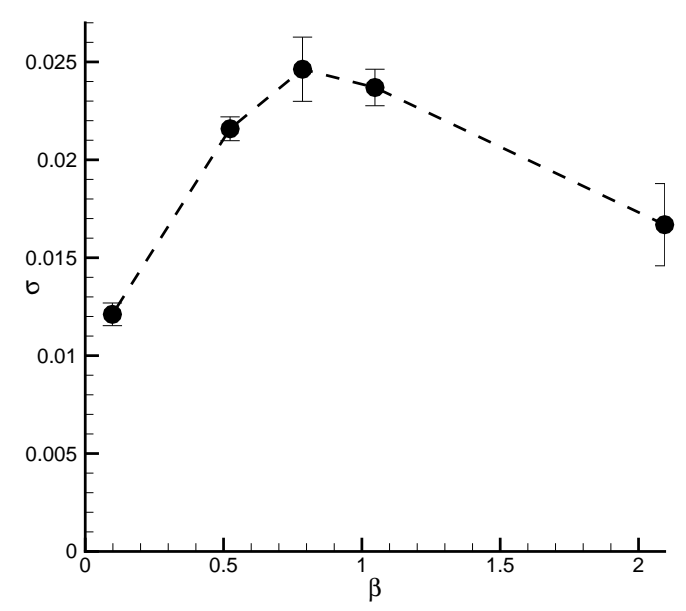

(b)

Figure 15. Spanwise variation for the jet in crossflow with different spanwise lengths: (a) spanwise variation growth with time, (b) spanwise variation growth rate with error bar.

The same kind of Gaussian pulse used for the ramp case is added to the baseflow above the jet slot centred at $(113,6)$ to study $3 \mathrm{D}$ instabilities. An exponential growth of spanwise variations appears after an initial development of about 300 in non-dimensional time. The growth rate of the instability varies with the spanwise wavelength, as shown in Figure 15(a) where the y-axis is displayed on a logarithmic scale. By measuring the slopes of the curves in the exponential stage, the variation of growth rate with wavenumber is obtained and plotted in Figure 15(b) with an estimated error bar. The error estimates shown on the Figure were obtained by computing the slopes at three different monitor points. The most unstable mode corresponds to the flow with a forcing wavelength of around 8 . This was confirmed by simulating a case with $L_{z}=64$ case with spanwise grid point $N_{z}=32$ where 7 spanwise periodic wave were observed all over the flowfield, corresponding to a most unstable wavelength of about 9 .

After increasing exponentially, the instability reaches a saturated stage. Figure 16 shows the distributions of $w$ velocity on different $y-z$ slices at different stages for a case with $L_{z}=6$ computed with $N_{z}=32$. Compared with the $w$ velocity distribution in the exponential growth stage on Figure 16(a), the amplitude of the $w$ velocity is increasing with time and higher harmonics appear as shown in Figure 16(b). All of the observations show the single harmonic structure of the disturbance breaks down at the saturated flow stage. The 3D instability appears to develop from edges of vortices in the upstream recirculation zone and above the jet. It is also observed that the upstream 3D instability starts and becomes saturated earlier than that in downstream flow. 


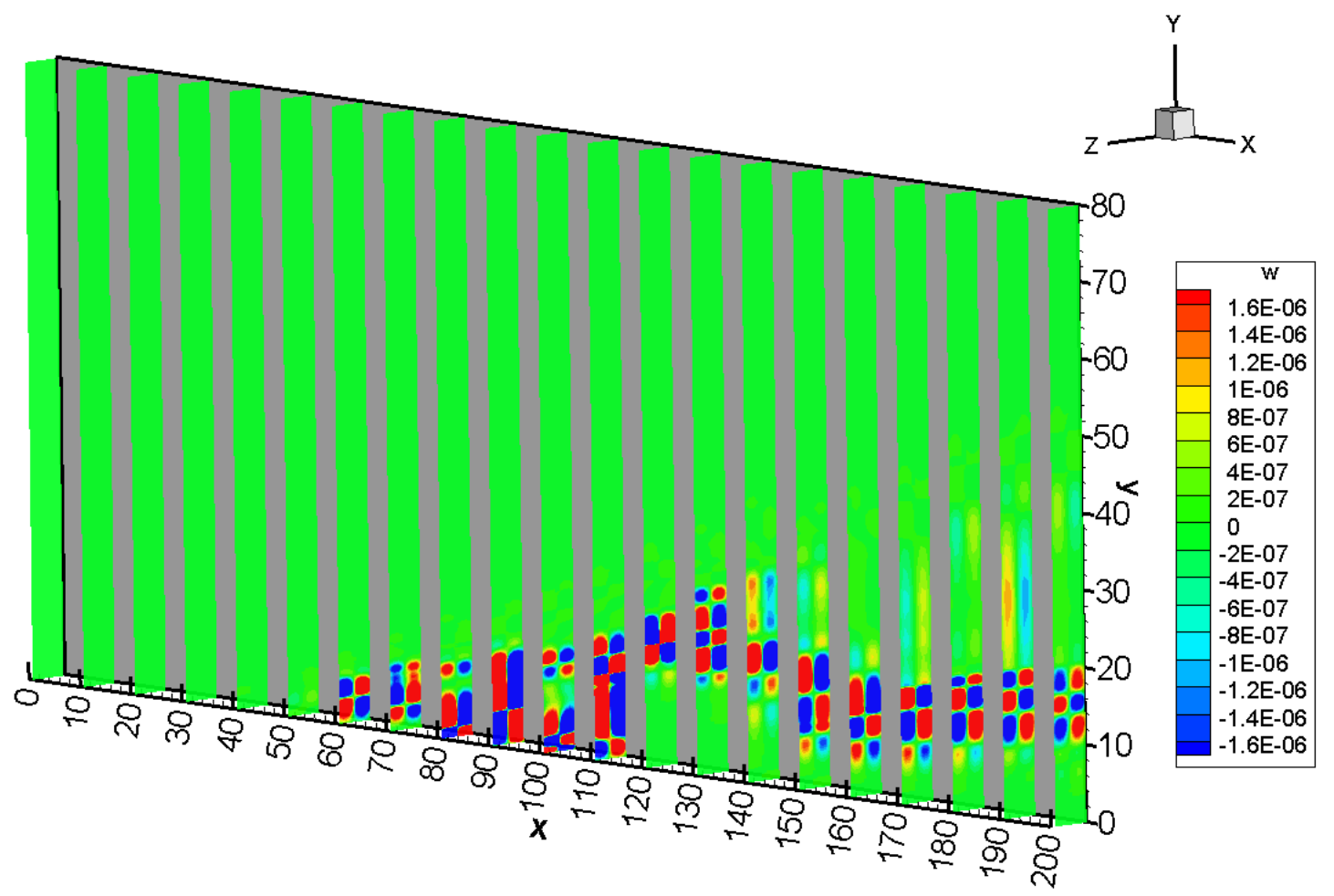

(a)

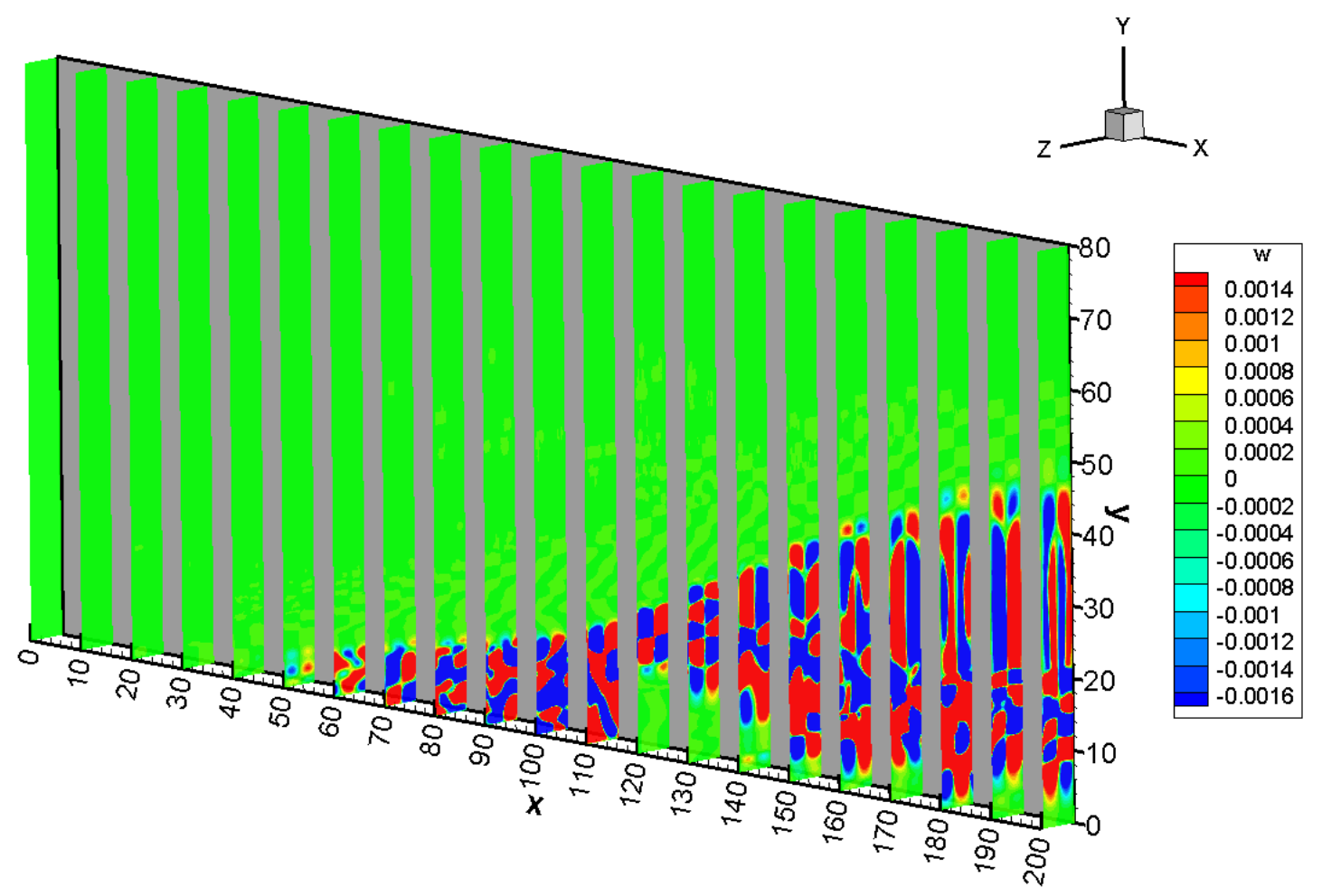

(b)

Figure 16. Distribution of $w$ velocity on $\mathbf{y z - s l i c e s}$ for $L_{z}=6$ case (a) at $\mathbf{t}=1600$ in exponential stage, (b) at $\mathbf{t}=\mathbf{2 4 0 0}$ in saturated stage. 
Compared with the uniform spanwise distribution seen for the streamwise velocity $u$ in the exponential stage, the variation of $u$ velocity in the saturated stage observed at $x=90$ and downstream shows the presence of streamwise vortices as shown in Figure 17(a). The vortices develop towards the wall but don't reach it as plotted in Figure 17(b) which shows contours of $u$ near the exit superimposed by streamlines. There are two pairs of counter-rotating vortices, depicted by the streamlines located above $y=10$.

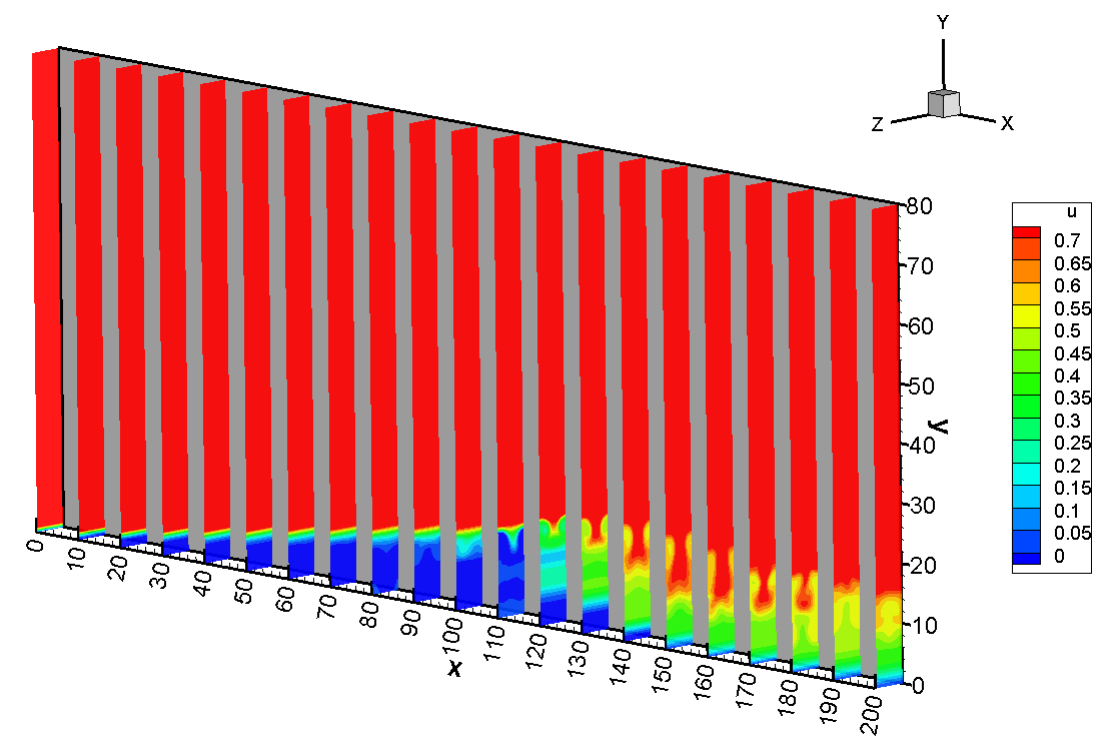

(a)

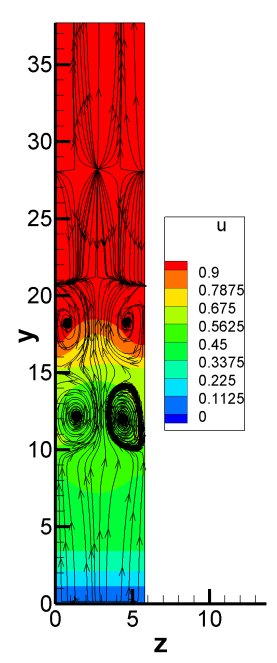

(b)

Figure 17. Contours of the streamwise velocity (a) on different yz-slices, (b) on $x=199.5$ plane.

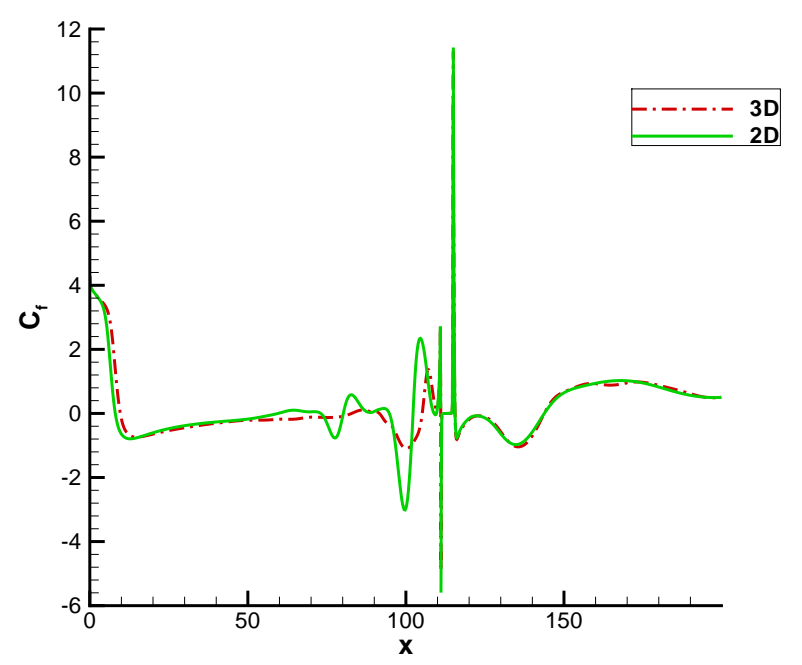

Figure 18. Friction coefficient distribution.

The 3D unstable flow caused by the jet in crossflow is analysed by plotting the skin friction coefficient $\left(C_{f}\right)$ distribution for the 2D steady flow and the 3D saturated flow as shown in Figure 18 . In the 3D flow, $C_{f}$ is averaged in the spanwise direction at one instantaneous time. It is found that the $x$ position where $C_{f}=0$, which corresponds to separation point, is affected by the $3 \mathrm{D}$ instability leading to a slightly shorter bubble. It can be seen that the variation in amplitude of $C_{f}$ upstream of the jet is smaller than that in saturated $2 \mathrm{D}$ flow. 


\section{Comparison of the separated flow for ramp and jet in crossflow}

Pagella ${ }^{9}$ stated that SWBLI between flows with ramp corner and impinging shock wave were practically identical for 2D flow. At the low Reynolds number used in their study, there was only one circulation zone with one large vortex for both ramp and shock wave impingement. For the ramp flow and the jet in crossflow studied here, larger Reynolds numbers and larger $J_{p}$ values for jet injection were used, causing large recirculation zones with a series of vortices. The recirculation zone of the jet in crossflow is divided into two regions by the jet, but similar recirculation zones are observed. The recirculation zones for the $2 \mathrm{D}$ ramp flow with a $12^{\circ}$ wedge angle for Reynolds number of 6,843 and the $2 \mathrm{D}$ jet in crossflow with $J_{p}$ of 0.3 are compared in Figure 19(a) and Figure 19(b). A similar series of secondary recirculation zones are found in both flows. They lie at the back of the main bubble for the ramp flow, while for the jet in crossflow they lie in front. By extracting the A-A sections shown in plots 19(a) and 19(b), it can be seen that the profiles shown in plots $19(\mathrm{c})$ and $19(\mathrm{~d})$ follow the same trend within the boundary layer. The jumps at $y=55$ in Figure 19(c) and at $y=15$ in Figure 19(d) are caused by separation shocks.

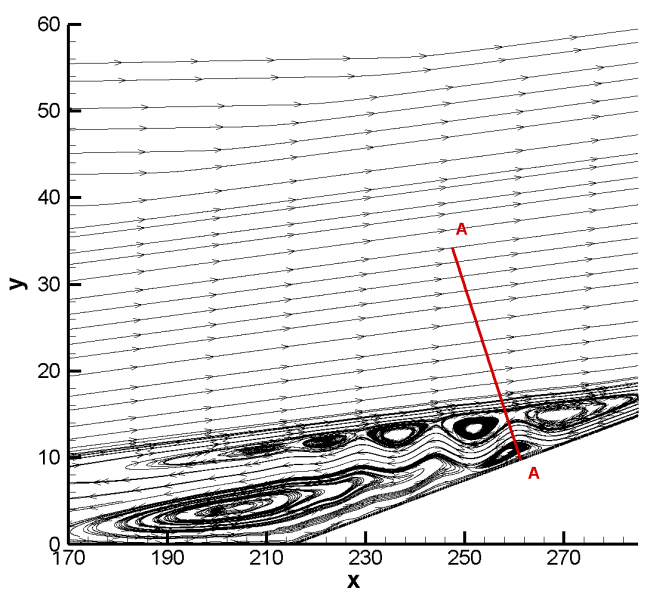

(a)

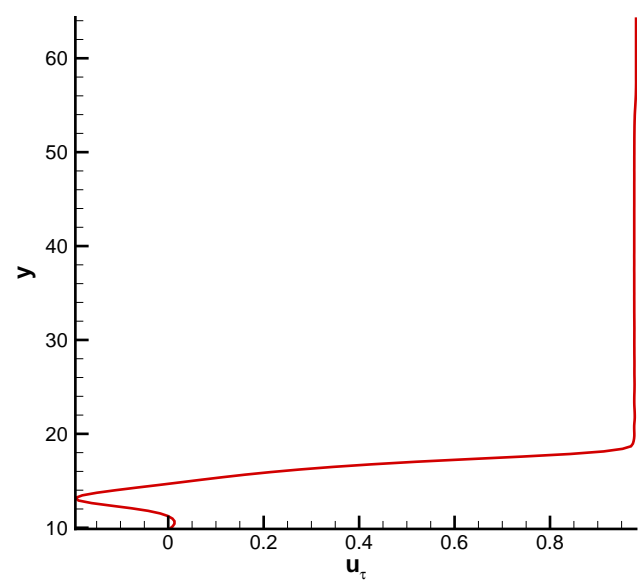

(c)

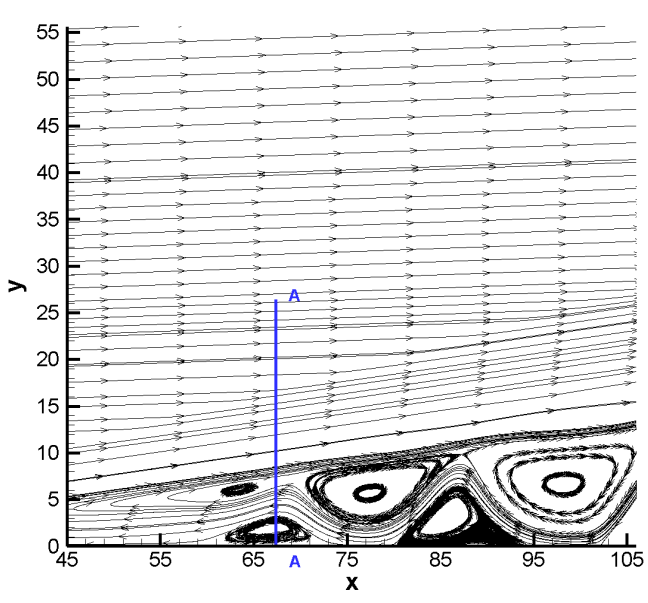

(b)

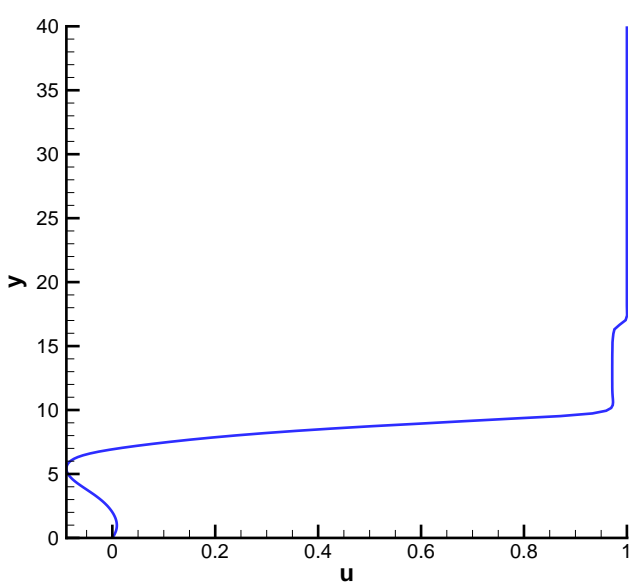

(d)

Figure 19. Comparison of the flow separations in the ramp and the jet cases: (a) recirculation zone in ramp flow, (b) recirculation zone in jet in crossflow, (c) profile of tangential velocity on AA line in ramp flow, (d) $u$ velocity profile on AA line in jet in crossflow. 


\section{Summary and future work}

Simulations of a supersonic ramp flow and a jet in supersonic crossflow have been conducted. Ramp flow was found to be globally unstable when the Reynolds number 6,843 was used, compared to a previous validation at $R e=3,422$ which was only convectively unstable. The jet in crossflow becomes unstable as $J_{p}$ is increased, with a critical value of $J_{p}$ between 0.1 and 0.3 . The most unstable mode for the ramp flow has a wavelength of 12, while the most unstable wavelength is 8 for jet in crossflow, based on the incoming boundary layer displacement thickness in both cases. Similar recirculation zones and wall-normal distributions of the streamwise velocity in these zones between ramp flow and jet in crossflow are observed. A string streamwise vortex is observed in the jet case in the saturated state formed after disturbance growth. The vortex is located away from the wall and does not have a strong effect on flow porperties at the wall.

\section{References}

${ }^{1}$ Dolling, D., "Fifty years of shock-wave/boundary-layer interaction research: What next?" AIAA Journal, Vol. 39, No. 8, Aug 2001, pp. 1517-1531.

${ }^{2}$ Robinet, J.-C., "Bifurcations in shock-wave/laminar-boundary-layer interaction: global instability approach," Journal of Fluid Mechanics, Vol. 579, May 2007, pp. 85-112.

${ }^{3}$ Adams, N., "Direct simulation of the turbulent boundary layer along a compression ramp at $M=3$ and $R e_{\theta}=1685$," Journal of Fluid Mechanics, Vol. 420, Oct 2000, pp. 47-83.

${ }^{4}$ Grilli, M., Schmid, P. J., Hickel, S., and Adams, N. A., "Analysis of unsteady behaviour in shockwave turbulent boundary layer interaction," Journal of Fluid Mechanics, Vol. 700, 2012, pp. 16-28.

${ }^{5}$ Loginov, M. S., Adams, N. A., and Zheltovodov, A. A., "LES of shock wave/turbulent boundary layer interaction," High Performance Computing in Science and Engineering, Vol. 3, 2006, pp. 222-234.

${ }^{6}$ Adams, N., "Direct numerical simulation of turbulent supersonic compression ramp flow," Computational Fluid Dynamics, Vol. 1, 1998, pp. 28-33.

${ }^{7}$ Marini, M., "Analysis of hypersonic compression ramp laminar flows under sharp leading edge conditions," Aerospace Science and Technology, Vol. 5, No. 4, Jun 2001, pp. 257-271.

${ }^{8}$ Pagella, A., Rist, U., and Wagner, S., "Numerical investigations of small-amplitude disturbances in a boundary layer with impinging shock wave at Ma=4.8," Physics of Fluid, Vol. 14, 2002, pp. 2088-2101.

${ }^{9}$ Pagella, A., Babucke, A., and Rist, U., "Two-dimensional numerical investigations of small-amplitude disturbances in a boundary layer at $\mathrm{Ma}=4.8$ : Compression corner versus impinging shock wave," Physics of Fluids, Vol. 16, No. 7, Jul 2004, pp. 2272-2281.

${ }^{10}$ Yao, Y., Krishnan, L., Sandham, N. D., and Roberts, G. T., "The effect of Mach number on unstable disturbances in shock/boundary-layer interactions," Physics of Fluids, Vol. 19, No. 5, May 2007, pp. 054104-1-054104-15.

${ }^{11}$ Lüdeke, H. and Sandham, N. D., "Direction numerical simulation of the transition process in a separated supersonic ramp flow," AIAA 2010-4470, 2010.

${ }^{12}$ Chanetz, B., Benay, R., Bousquet, J.-M., Bur, R., Pot, T., Grasso, F., and Moss, J., "Experimental and numerical study of the laminar separation in hypersonic flow," Aerospace Science and Technology, Vol. 2, No. 3, 1998, pp. $205-218$.

${ }^{13}$ Robert W. Cubbison, Bernhard H. Anderson, J. J. W., "Surface Pressure Distributions with a Sonic Jet Normal to Adjacent Flat Surfaces at Mach 2.92 to 6.4," Tech. Rep. NASA TN D-580, 1961.

${ }^{14}$ Kaufman II, L. G., "Hypersonic flows past transverse jets," Journal of Spacecraft, Vol. 4(9), 1967, pp. 1230-1235.

${ }^{15}$ Lee, C. C. and Barfield, B. F., "Interaction of sonic transverse jets with supersonic external flows," AIAA Journal, Vol. 9, No. 2, 1971, pp. 304-308.

${ }^{16}$ Powrie, H., A study of the interaction between an underexpanded normal jet and a hypersonic freestream, Ph.D. thesis, School of Engineering Sciences, University of Southampton, 1996.

${ }^{17}$ Roberts, G., Schuricht, P., and Mudford, N., "Heating enhancement caused by a transverse control jet in hypersonic flow," Shock Waves, Vol. 8, 1998, pp. 105-112.

${ }^{18}$ Qin, N. and Redlich, A., "Massively separated flows due to transverse sonic jet in laminar hypersonic stream," Shock Waves, Vol. 9, 1999, pp. 87-93.

${ }^{19}$ Gajbhiye, R. R., "Unsteady aspect of control jets for high speed applications," MSc Thesis, School of Engineering Sciences, University of Southampton, 2010.

${ }^{20}$ Ilak, M., Schlatter, P., Bagheri, S., and Henningson, D. S., "Bifurcation and stability analysis of a jet in cross-flow: onset of global instability at a low velocity ratio," Journal of Fluid Mechanics, Vol. 696, 2012, pp. 94-121.

${ }^{21}$ Carpenter, M., Nordstrom, J., and Gottlieb, D., "A stable and conservative interface treatment of arbitrary spatial accuracy," Journal of Computational Physics, Vol. 148, No. 2, Jan 1999, pp. 341-365.

${ }^{22}$ Wray, A. A., "Very low storage time-advancement schemes," Internal Report, 1986.

${ }^{23}$ Sandham, N. D., Li, Q., and Yee, H., "Entropy splitting for high-order numerical simulation of compressible turbulence," Journal of Computational Physics, Vol. 178, No. 2, May 2002, pp. 307-322.

${ }^{24}$ Yee, H., Sandham, N., and Djomehri, M., "Low-dissipative high-order shock-capturing methods using characteristic-based filters," Journal of Computational Physics, Vol. 150, No. 1, Mar 1999, pp. 199-238.

${ }^{25}$ Ducros, F., Ferrand, V., Nicoud, F., Weber, C., Darracq, D., Gacherieu, C., and Poinsot, T., "Large-Eddy Simulation of the Shock/Turbulence Interaction," Journal of Computational Physics, Vol. 152, No. 2, 1999, pp. 517 - 549. 
${ }^{26}$ Harten, A., "The artificial compression method for computation of shocks and contact discontinuities. III. Self-adjusting hybrid schemes," Mathematics of Computation, Vol. 32, No. 142, 1978, pp. 363-389.

${ }^{27}$ White, F. M., Viscous Fluid Flow, McGraw-Hill, 1974. 